\title{
ON EQUINORMAL QUASI-METRICS
}

\author{
by SALVADOR ROMAGUERA
}

(Received 26th July 1987)

Throughout this paper all spaces are $T_{1}$ and $N$ will denote the set of all positive integer numbers.

A quasi-metric on a set $X$ is a non-negative real-valued function $d$ on $X \times X$ such that, for all $x, y, z \in X$, (i) $d(x, y)=0$ if, and only if, $x=y$; (ii) $d(x, y) \leqq d(x, z)+d(z, y)$.

The topology $\tau(d)$ induced on $X$ by a quasi-metric $d$ has as a base the family of $d$ balls $\left\{B_{d}(x, r): x \in X, r>0\right\}$ when $B_{d}(x, r)=\{y \in X: d(x, y)<r\}$. A space $(X, \tau)$ is quasimetrizable if there exists a quasi-metric $d$ on $X$ such that $\tau=\tau(d)$.

If $d$ is a quasi-metric on $X$, let $d^{-1}(x, y)=d(y, x)$ for all $x, y \in X$. Then $d^{-1}$ is also a quasi-metric on $X$. A quasi-metric $d$ on $X$ is called strong [13] if $\tau(d) \subset \tau\left(d^{-1}\right)$. We say that a quasi-metric $d$ is equinormal [9] if $d(A, B)>0$ for every two disjoint closed sets $A$ and $B$ of $X$. In a similar way one defines the notion of equinormal metric [11] and equinormal $\gamma$-metric whenever $d$ is, respectively, a metric or a $\gamma$-metric. We say that a space $(X, \tau)$ admits an equinormal (metric, $\gamma$-metric) quasi-metric if there exists an equinormal (metric, $\gamma$-metric) quasi-metric $d$ on $X$ such that $\tau=\tau(d)$.

Equinormal quasi-metrics are an interesting class of strong quasi-metrics. Fletcher and Lindgren have proved $[9,1]$, that a Hausdorff space admits an equinormal metric if it admits an equinormal quasi-metric. They also have proved that every equinormal quasi-metric is complete. On the other hand since a quasi-metrizable space is compact if, and only if, every compatible quasi-metric is strong [8], we deduce that a quasimetrizable space is compact if, and only if, every compatible quasi-metric is equinormal. The purpose of this note is to give some necessary and sufficient conditions in order that a space admits an equinormal quasi-metric. Furthermore we deduce that equinormal quasi-metrics are invariant under continuous closed mappings.

Terms and concepts which are not defined here may be found in [1] and [4].

Proposition 1. A space $(X, \tau)$ admits an equinormal quasi-metric if, and only if, it has a decreasing sequence $\left\{U_{n}: n \in N\right\}$ of neighbournets such that for every two disjoint closed sets $A$ and $B$ of $X$ there exists a $k \in N$ satisfying $U_{k}^{2}(A) \cap B=\varnothing$.

Proof. Sufficient condition. It is clear that $(X, \tau)$ is a $\gamma$-space by means of the sequence $\left\{U_{n}: n \in N\right\}$. Let now $x \in U_{n}\left(x_{n}\right)$ for all $n \in N$, and suppose that $\left\{x_{n}: n \in N\right\}$ is not convergent to $x$; then there exists a subsequence $\left\{x_{n m}: m \in N\right\}$ and a closed set $A$ such 
that $\left\{x_{n m}: m \in N\right\} \subset A$ and $x \in X-A$. Because $U_{j}^{2}(A) \cap\{x\}=\varnothing$ for some $j \in N$, we have a contradiction. Therefore $(X, \tau)$ is a semi-stratifiable space and, hence, it is developable [3]. According Fox's lemma [2] there exist neighbournets $\tilde{U}_{1}$ and $V_{1}$ satisfying $\tilde{U}_{1}^{4} \subset U_{1}^{2}$ and $V_{1}^{4} \subset \tilde{U}_{1}^{2}$, this is $V_{1}^{6} \subset U_{1}^{2}$. Similarly, we obtain, for all $n>1$, a neighbournet $V_{n}$ satisfying $V_{n}^{6} \subset\left(V_{n-1} \cap U_{n}\right)^{2}$. If we put $W_{n}=V_{n}^{2}$ then $W_{n}^{3} \subset W_{n-1}$ for all $n>1$ and by Kelley's lemma [5, page 185] there exists a quasi-metric $d$ on $X$ such that

$$
W_{n} \subset\left\{(x, y): d(x, y)<2^{-n}\right\} \subset W_{n-1}
$$

for all $n>1$. Because $W_{n}^{3} \subset U_{n}^{2}$ then $\tau=\tau(d)$. Finally, if $A$ and $B$ are disjoint closed sets of $X$ we have $U_{k}^{2}(A) \cap B=\varnothing$ for some $k \in N$. Consequently, $d(A, B) \geqq 2^{-(k+1)}$ and, hence, $d$ is equinormal.

Necessary condition. It is enough to take, for each $n \in N, U_{n}=\{(x, y): d(x, y)<1 / n\}$ whenever $d$ is an equinormal quasi-metric on $X$ such that $\tau=\tau(d)$.

In [9] Lindgren and Fletcher introduce the notion of uniform $D_{1}$ space and prove its relevance in the study of equinormal metrics. A space $(X, \tau)$ is uniform $D_{1}$ if there exists a function $g: N \times X \rightarrow \tau$ such that for each closed set $F$ the family $\left\{W_{n}(F): n \in N\right\}$ is a base for $F$ where $W_{n}(F)=\cup\{g(n, x): x \in F\}$. In this case we say that $g$ is a uniform $D_{1}$ function. The following result shows that uniform $D_{1}$ spaces also play an important role in characterizing all spaces which admit an equinormal quasi-metric.

Theorem 1. A space $(X, \tau)$ admits an equinormal quasi-metric if, and only if, it is a uniform $D_{1}$ space and a $\gamma$-space.

Proof. Let $d$ be a $\gamma$-metric [7] on $X$ such that $\tau(d)=\tau$ and let $g$ be a uniform $D_{1}$ function for $(X, \tau)$. It is not a restriction to suppose $g(n+1, x) \subset g(n, x)$ for each $x \in X$ and each $n \in N$.

Let now, for each $x \in X$ and each $n \in N, U_{n}(x)=B_{d}(x, 1 / n) \cap g(n, x)$. Suppose that $A$ and $B$ are disjoint closed sets satisfying $U_{k}^{2}(A) \cap B \neq \varnothing$ for all $k \in N$. Then there exist sequences $\left\{a_{k}: k \in N\right\},\left\{b_{k}: k \in N\right\}$ and $\left\{y_{k}: k \in N\right\}$ such that $a_{k} \in A, b_{k} \in B, y_{k} \in U_{k}\left(a_{k}\right)$ and $b_{k} \in U_{k}\left(y_{k}\right)$ for all $k \in N$. We first note that the sequence $\left\{a_{k}: k \in N\right\}$ has no accumulation point since, in the other case, taking into account that $d$ is a $\gamma$-metric we obtain $A \cap B \neq \varnothing$. It is also clear that there exists a $k_{0} \in N$ such that $G \cap A=\varnothing$ when $G=\left\{y_{k}: k \geqq k_{0}\right\}$. If there exists some $a_{m} \in \bar{G}-G$ we have $a_{m} \in B$, a contradiction. Consequently, $a_{k} \in X-\bar{G}$ for all $k \in N$, and, hence, there exists $i \geqq k_{0}$ such that $\left(\cup\left\{g\left(i, a_{k}\right): k \in N\right\}\right) \cap \bar{G}=\varnothing$ which is impossible since $y_{i} \in g\left(i, a_{i}\right)$. From Proposition 1, $(X, \tau)$ admits an equinormal quasi-metric. The converse is obvious.

Corollary. A space $(X, \tau)$ admits an equinormal quasi-metric if, and only if, it admits an equinormal $\gamma$-metric.

In [6] Kofner proves that continuous closed mappings with first countable range 
preserve quasi-metrizable spaces. Künzi has shown that continuous closed mappings with first countable range preserve strongly quasi-metrizable spaces [8]. In this direction we have:

Theorem 2. Let $f$ be a continuous closed mapping from a space $(X, \tau)$ onto a space $\left(Y, \tau^{\prime}\right)$. If $(X, \tau)$ admits an equinormal quasi-metric then $\left(Y, \tau^{\prime}\right)$ admits an equinormal quasi-metric.

Proof. In [10] it is proved that continuous pseudo-open mappings preserve uniform $D_{1}$ spaces. Hence $\left(Y, \tau^{\prime}\right)$ is a uniform $D_{1}$ space and, by Kofner's theorem, it is quasi-metrizable. Theorem 1 concludes the proof.

Corollary (Rainwater [12]). Let $f$ be a continuous closed mapping from a space $(X, \tau)$ onto a space $\left(Y, \tau^{\prime}\right)$. If $(X, \tau)$ admits an equinormal metric then $\left(Y, \tau^{\prime}\right)$ admits an equinormal metric.

Proof. $\left(Y, \tau^{\prime}\right)$ is a Hausdorff space which admits an equinormal quasi-metric by virtue of Theorem 2. The conclusion follows from [9, Proposition 4.1].

The author like to express his gratitude to Dr H. P. Künzi because his article [8] suggested several ideas of this paper.

\section{REFERENCES}

1. P. Fletcher and W. F. Lindgren, Quasi-uniform Spaces (Marcel Dekker, New York, 1982).

2. R. Fox, A short proof of the Junnila quasi-metrization theorem, Proc. Amer. Math. Soc. 83 (1981), 663-664.

3. R. E. Hodel, Spaces defined by sequences of open covers which guarantee that certain sequences have cluster points, Duke Math. J. 39 (1972), 253-263.

4. H. J. K. JunNila, Neighbornets, Pacific J. Math. 76 (1978), 83-108.

5. J. L. Kelley, General Topology (Springer-Verlag, New York. 1955).

6. J. Kofner, Closed mappings and quasi-metrics, Proc. Amer. Math. Soc. 80 (1980), 333-336.

7. J. Kofner, Transitivity and ortho-bases, Canad. J. Math. 33 (1981), 1439-1447.

8. H. P. Künzi, On strongly quasi-metrizable spaces, Arch. Math. (Basel) 41 (1983), 57-63.

9. W. F. LindGREN and P. Fletcher, Equinormal quasi-uniformities and quasi-metrics, Glasnik Mat. 13 (1978), 111-125.

10. J. A. Martin-Alustiza and S. Romaguera, Pseudo-open mappings on $D_{1}$ spaces, Topology conference (Proceedings X Jornadas Hispano-Lusas de matemáticas. Univ. Murcia 1985), 66-71 (Spanish).

11. S. G. MrówKa, On normal metrics, Amer. Math. Monthly 72 (1965), 998-1001.

12. J. Rainwater, Spaces whose finest uniformity is metric, Pacific J. Math. 9 (1959), 567-570. 
13. R. A. Stoltenberg, On quasi-metric spaces, Duke Math. J. 36 (1969), 65-72.

Departamento de Matemáticas

ETSICCP

UNIVERSIDAD POLITÉCNICA

46071 VALENCIA

SPAIN 